Ann. Biol. anim. Bioch. Biophys., I963, 3 (4), 433-435

\title{
ALLÈLES DU LOCUS S DE GROUPES SANGUINS DES BOVINS
}

\author{
NOUVEAUX RÉsultats
}

F. GROSCLALDE:

Aver la collaboration technique de Anne Ir Girasis et G. BÉzaro

Laboratoire des Groupes sanguins,

Centre national de Recherches zootechniques, Jouy-en-Josas (Seine-et-Oise)

\section{SOMMAIRE}

Un nouvel anticorps, anti- $\mathrm{S}^{\prime \prime}$, permet de subdiviser l'ancien phénogroupe $\mathrm{S}_{1} \mathrm{II}^{\prime}$ en deux nouveaux phénogroupes (1) $: S_{1} \mathrm{~S}^{\prime \prime} \mathrm{H}^{\prime}$ et $\mathrm{S}_{1} \mathrm{H}^{\prime}$; par ailleurs, anti- $\mathrm{S}^{\prime \prime}$ permet de distinguer un phénogroupe $\mathrm{S}^{\prime \prime} \mathrm{H}^{\prime}$, rencontré juscuu'à présent dans les races Normande et Charolaise, où il est assez rare.

Ceci porte à to le nombre d'allèles détectés au locus $S$ dans notre laboratoire; cependant, des données partielles sur diverses races en cours d'étude, indiqutent la présence d'autres allèles, détectables à l'aide des réactifs décrits jusqu'ici.

\section{INTRODUC'TION}

Dans un travail récent, (Grosclaude et Millot, I963), nous décrivions notamment deux nouveaux anticorps primaires relatifs au système $S$ de groupes sanguins des bovins, anti- $\mathrm{U}^{\prime \prime}$ et anti- $\mathrm{U}_{\mathrm{z}}^{\beta}$, qui permettent de distinguer 8 allèles à ce locus, au lieu de 5 précédemment.

La présente note, qui complète ces résultats, a pour but de décrire un autre anticorps, anti- $\mathrm{S}^{\prime \prime}$, permettant de porter à ro le nombre d'allèles détectés au locus $\mathrm{S}$ dans notre laboratoire.

\section{TECHNIQUES ET MÉTHODES}

Les techniques et méthodes utilisées ont été résumées dans notre précédente publication (GrosCLAUdE et MLLLOT, 1963).

(1) Xous rappelons que le terme de phénogroupe, introduit par Stormont (1955), désigne un groupe de facteurs antigéniques commandé par un gène. Îl faut en effet distinguer les gènes - ou allèles - d'une part, et les facteurs antigéniques qu'ils déterminent, associés en phénogroupes, d'autre part. 


\section{RÉSULTATS}

Dans le travail cité ci-dessus, nous mentionnions que le bovin $\mathrm{N}^{\circ} \mathrm{I} 46 \mathrm{I}$, de race Abondance, et de phénotype $\mathrm{H}^{\prime}$ au locus $\mathrm{S}$, immunisé par des hématies de phénotype $\mathrm{S}_{1} \mathrm{H}^{\prime}$, avait produit une population de quatre anticorps relatifs à ce système $S$ : anti- $\mathrm{S}_{1}$, anti- $\left(\mathrm{S}_{1} \mathrm{U}^{\prime}\right)$, anti- $\left(\mathrm{S}_{1} \mathrm{U}^{\prime} \mathrm{U}_{1}\right)$, anti- $\left(\mathrm{S}_{1} \mathrm{U}^{\prime \prime}\right)$. Or nous avons constaté ultérieurement qu'une cinquième fraction, contenue dans le sérum de ce bovin, était également relative au système $S$; ce nouvel anticorps a été dénommé anti- $S^{\prime \prime}$.

Nos observations concernant le facteur $S^{\prime \prime}$, nous ont amené aux conclusions suivantes:

- $\mathrm{S}^{\prime \prime}$ subdivise l'ancien phénogroupe $\mathrm{S}_{1} \mathrm{H}^{\prime}$ en deux nouveaux phénogroupes, $S_{1} S^{\prime \prime} H^{\prime}$ et $S_{1} H^{\prime}$ : du fait de l'existence du facteur $S^{\prime \prime}$, l'appellation $S_{1} H^{\prime}$ a une autre signification que précédemment.

- $S^{\prime \prime}$ appartient bien au système $S$, car, dans plus de I50 filiations comportant un parent hétérozygote pour le facteur $S_{1}$ et pour le facteur $S^{\prime \prime}$, et l'autre parent doublement négatif, $S_{1}$ et $S^{\prime \prime}$ restent toujours associés : ils sont transmis ensemble, ou non transmis tous les deux.

- Les spécificités des deux facteurs sont différentes: des hématies $S_{1}$ non $S^{\prime \prime}$ absorbent du sérum I46r tous les anticorps du système $S$, sauf anti- $S^{\prime \prime}$, dont le titre reste inchangé.

-- Outre les phénogroupes $S_{1} S^{\prime \prime} H^{\prime}$ et $S_{1} H^{\prime}$, il existe un phénogroupe $S^{\prime \prime} H^{\prime}$; ce phénogroupe est plus rare : nous l'avons observé jusqu'ici en race Charolaise et en race Normande : dans cette dernière nous avons pu étudier la descendance d'un taureau possédant ce phénogroupe, pour vérifier qu'il comportait le facteur $\mathrm{H}^{\prime}$.

I.es relations existant entre les I I anticorps et Io phénogroupes du système $S$ actuellement connus dans notre laboratoire sont alors les suivantes : (tabl. I).

\section{TABLEAU I}

Relations existant entre les I I anticorps et Io phénogroupes du système $S$ de groupes sanguins des bonins : le signe + indique une réaction positive

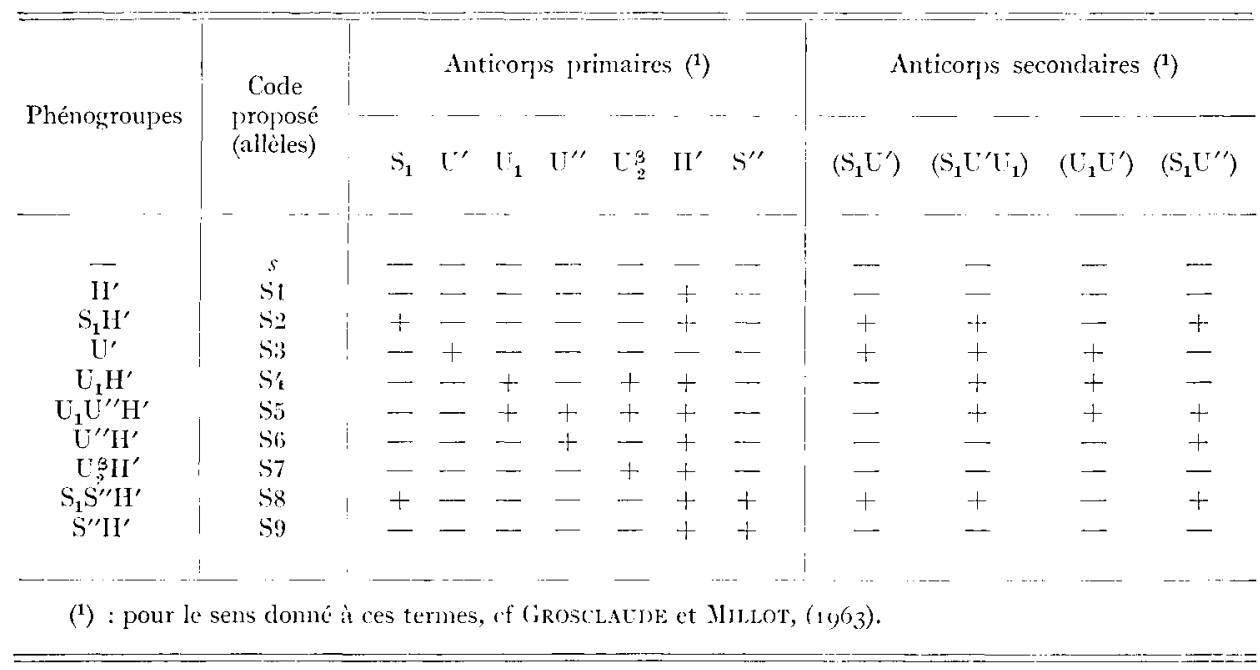




\section{DISCUSSION}

Il existe une certaine analogie entre les couples de facteurs $\mathrm{U}_{1}$ et $\mathrm{U}^{\prime \prime}$ d'une part, et $S_{1}$ et $S^{\prime \prime}$ d'autre part; en effet les trois combinaisons possibles entre $U_{1}$ et $U^{\prime \prime}$ ont été observées $\left(\mathrm{U}_{1} \mathrm{H}^{\prime}, \mathrm{U}_{1} \mathrm{U}^{\prime \prime} \mathrm{H}^{\prime}, \mathrm{U}^{\prime \prime} \mathrm{H}^{\prime}\right)$, de même que les trois combinaisons entre $S_{1}$ et $S^{\prime \prime}\left(S_{1} H^{\prime}, S_{1} S^{\prime \prime} H^{\prime}, S^{\prime \prime} H^{\prime}\right)$; en revanche, nous n'avons trouvé jusqu'à présent aucun phénogroupe formé de facteurs pris dans les deux couples.

Dans notre précédent travail, cité ci-dessus, nous avions donné à l'allèle déterminant le phénogroupe $S_{1} H^{\prime}$ le numéro de code $S_{2}$; ce phénogroupe a été subdivisé par le facteur $S^{\prime \prime}$ : nous avons conservé 1'appellation $\mathrm{S} 2$ pour 1'allèle déterminant le phénogroupe $S_{1} H^{\prime}$, et donné la dénomination $S 8$ à l'allèle déterminant $S_{1} S^{\prime \prime} H^{\prime}$.

Comme élément de différenciation des races, $U^{\prime \prime}$ semble supérieur à $S^{\prime \prime}$ puisqu'il est très rare dans certaines (GROScIAUDE et MIL, I, T, I963), alors que $S^{\prime \prime}$ est assez également réparti; par contre, comme élément de différenciation des génotypes sanguins, $\mathrm{S}^{\prime \prime}$ est alors supérieur à $\mathrm{U}^{\prime \prime}$ : dans la race Frisonne par exemple, si l'allèle S2 est le plus répandu, S8 a néanmoins une fréquence non négligeable : l'utilisation d'anti- $\mathrm{S}^{\prime \prime}$ est donc avantageuse dans cette race.

Avec ro allèles, 1e locus S devient, à égalité avec le locus A, (STORmont, I962), le troisième locus par ordre de variabilité décroissante, après les loci $B$ et $C$; cependant, comme l'analyse génétique de ce dernier est souvent difficile par suite de la fréquence élevée de plusieurs facteurs, le locus $S$ offre, dans certaines races françaises, plus de possibilités pour les études et contrôles génétiques.

En outre, des données partielles sur certaines races de la moitié sud de la France, (Salers, Blonde d'Aquitaine), révèlent une variabilité encore plus forte au locus $\mathrm{S}$, où 2 ou 3 allèles supplémentaires au moins seraient présents.

Rę̧u pour publication en novembre $19^{6} 3$.

\section{SUMMARY \\ ALLELES OF THE S SYSTEM OF BOVINE BLOOD-GROUPS; NEW RESULTS}

A new antibody, called anti- $\mathrm{S}^{\prime \prime}$, subdivides the late phenogroup $\mathrm{S}_{\mathbf{1}} \mathrm{H}^{\prime}$ into two new phenogroups $\mathrm{S}_{1} \mathrm{~S}^{\prime \prime} \mathrm{H}^{\prime}$ and $\mathrm{S}_{1} \mathrm{H}^{\prime}$; moreover, anti-S" detects a phenogrowp $\mathrm{S}^{\prime \prime} \mathrm{H}^{\prime}$, encountered, up to now, in the Normande and Charolaise breeds, where it is rather rare.

This brings to to the number of alleles of the $\mathrm{S}$ system known in our laboratory; however, partial data on several breeds at present under study, indicate the existence of other alleles, distinguishable with reagents already described.

\section{RÉFÉRENCES BIBLIOGRAPHIQUES}

Groscladde F., Miliot, P., 1963. Alleles supplémentaires au locus $S$ de groupes sanguins des bovins. Ann. Biol. Anim. Bioch. Biophys., 3, I I9-124.

Stormont, C., 1955. Linked genes pseudoalleles and blood groups. Am. Naturalisl, 89, 105-I 16.

Stormont, C., ig62. Current status of blood groups in cattle. Ann. Y. Y. Acad. Sci., 97, $25^{1-268 .}$ 